\title{
Novel Insights of Effects of Pregabalin on Neural Mechanisms of Intracortical Disinhibition in Physiopathology of Fibromyalgia: An Explanatory, Randomized, Double-Blind Crossover Study
}

\author{
Alícia Deitos ${ }^{1}$, Matheus Dorigatti Soldatelli1,2, Jairo Alberto Dussán-Sarria1, \\ Andressa Souza ${ }^{2,3}$, Iraci Lucena da Silva Torres ${ }^{4}$, Felipe Fregni ${ }^{5,6}$ and \\ Wolnei Caumo ${ }^{1,2,7,8 *}$
}

\begin{abstract}
' Post-Gradaution in Medical Science at Medical School, Universidade Federal do Rio Grande do Sul, Porto Alegre, Brazil, ${ }^{2}$ Laboratory of Pain and Neuromodulation, Universidade Federal do Rio Grande do Sul, Porto Alegre, Brazil, ${ }^{3}$ La Salle University Center, Canoas, Brazil, ${ }^{4}$ Pharmacology Department, Instituto de Ciências Básicas da Saúde, Universidade Federal do Rio Grande do Sul, Porto Alegre, Brazil, ${ }^{5}$ Department of Neurology, Harvard Medical School, Boston, MA, United States, ${ }^{6}$ Berenson-Allen Center for Noninvasive Brain Stimulation, Department of Neurology, Beth Israel Deaconess Medical Center, Harvard Medical School, Boston, MA, United States, ${ }^{7}$ Anesthesiologist, Pain and Palliative Care Service, Hospital de Clínicas de Porto Alegre, Porto Alegre, Brazil, ${ }^{8}$ Surgery Department, School of Medicine, Universidade Federal do Rio Grande do Sul, Porto Alegre, Brazil
\end{abstract}

OPEN ACCESS

Edited by: Filippo Brighina Università degli Studi di Palermo, Italy

Reviewed by: Gianluca Coppola, Fondazione GB Bietti (IRCCS), Italy Yelena Granovsky, Rambam Health Care Campus, Israel

*Correspondence: Wolnei Caumo wcaumo@hcpa.edu.br

Received: 13 June 2018 Accepted: 20 September 2018 Published: 19 November 2018

Citation:

Deitos A, Soldatelli MD, Dussán-Sarria JA, Souza A, da Silva Torres IL, Fregni $F$ and Caumo W (2018) Novel Insights of Effects of Pregabalin on Neural

Mechanisms of Intracortical Disinhibition in Physiopathology of

Fibromyalgia: An Explanatory, Randomized, Double-Blind Crossover Study. Front. Hum. Neurosci. 12:406. doi: 10.3389/fnhum.2018.00406
Background: The fibromyalgia (FM) physiopathology involves an intracortical excitability/inhibition imbalance as measured by transcranial magnetic stimulation measures (TMS). TMS measures provide an index that can help to understand how the basal neuronal plasticity state (i.e., levels of the serum neurotrophins brain-derived neurotrophic factor (BDNF) and S100-B protein) could predict the effect of therapeutic approaches on the cortical circuitries. We used an experimental paradigm to evaluate if pregabalin could be more effective than a placebo, to improve the disinhibition in the cortical circuitries in FM patients, than in healthy subjects (HS). We compared the acute intragroup effect of pregabalin with the placebo in FM patients and healthy subjects (HS) on the current silent period (CSP) and short intracortical inhibition (SICI), which were the primary outcomes. Pain scores and the pain pressure threshold (PPT) were secondary outcomes.

Methods: This study included 27 women (17 FM and $10 \mathrm{HS}$ ), with ages ranging from 19 to 65 years. In a blinded, placebo-controlled clinical trial, participants were randomized to receive, in a cross-over manner, oral pregabalin of $150 \mathrm{mg}$ or a placebo. The cortical excitability pain measures were assessed before and 90 min after receiving the medication.

Results: A generalized estimating equation (GEE) model revealed that in FM, pregabalin increased the CSP by $14.34 \%$ [confidence interval (Cl) 95\%; 4.02 to 21.63] and the placebo reduced the CSP by $1.58 \%(\mathrm{Cl} 95 \% ;-57$ to 25.9$)(P=0.00)$. Pregabalin reduced the $\mathrm{SICl}$ by $8.82 \%(\mathrm{Cl} 95 \%,-26$ to 46.00$)$ and the placebo increased it 
by $19.56 \%$ ( $\mathrm{Cl} 95 \% ; 8.10$ to $59.45 ; P=0.02$ ). Pregabalin also improved the pain measures. In the treatment group, the BDNF-adjusted index was positively correlated and the serum S100-B negatively correlated with the CSP, respectively. However, in the $\mathrm{HS}$, pregabalin and the placebo did not induce a statistically significant effect in either intracortical excitability or pain measures.

Conclusion: These results suggest that pregabalin's effect on cortical neural networks occurs, particularly under basal neuronal hyperexcitability, because its impact on the cortical excitability and the pain measures was observed only in the FM group. This indicates that pregabalin increased the CSP to induce inhibition in specific neural networks, while it increased the SICI to improve the excitability in other neurobiological systems. Trial registration in clinicaltrials.gov Identifier: NCT02639533.

Keywords: fibromyalgia, cortical silent period, short intracortical inhibition, BDNF, S100B

\section{INTRODUCTION}

Fibromyalgia (FM) is a syndrome that comprises of chronic widespread musculoskeletal pain, depressive symptoms, fatigue, sleep disturbance, and disturbances of the biological rhythm (Wolfe et al., 1990; Mease et al., 2005). Although the etiology of FM remains elusive, an appealing hypothesis is related to the overall hyperexcitement of the neurons based on central sensitization (CS) (Yunus, 2007; Latremoliere and Woolf, 2009; Woolf, 2011). The CS comprises a state that the central nervous system amplifies the sensory inputs in consequence to reinforcement on synaptic connectivity by structural and functional changes on axonic and dendritic terminals. The brain-derived neurotrophic factor (BDNF) plays a crucial role in these neuroplastic changes, and thus to the development and sustainment of CS pain. The FM is a CS syndrome in which patients have elevated serum levels of either S100-B protein or BDNF, which have been correlated with lower pain thresholds (Zanette et al., 2014). Additionally, in chronic pain, the increase in serum BDNF is associated with higher motor cortex disinhibition, indicated by a shorter cortical silent period (CSP) (Caumo et al., 2016).

In the clinical setting, the different individual symptoms and mechanisms of each CSP require a customized treatment approach. For this, we need to advance our understanding of alterations of neuroplasticity disease-related in vivo. A tool that provides insight to the neurotransmitter system, when we assess the cortical excitability, is transcranial magnetic stimulation (TMS) measures (Caipa et al., 2018). For example, FM patients, compared to healthy subjects (HS), showed a decrease in either short intracortical inhibition (SICI) or the CSP (Salerno et al., 2000). Since both SICI and CSP are mediated by inhibitory gamma-aminobutyric acid (GABAergic) interneurons within the primary motor cortex (Di Lazzaro et al., 2006; McDonnell et al., 2006), these findings have been interpreted as an indication for impaired GABA-mediated function in FM. Such metaplastic alterations were correlated with depression, catastrophizing, and fatigue scores in FM patients (Galhardoni et al., 2015).

As previously mentioned, the most clinical symptoms of FM have been linked to a defective inhibitory function. Pregabalin is a US Food and Drug Administration (FDA) approved drug for FM. According to in vitro studies, pregabalin exerts pharmacologic activity through direct interactions to a ligand of the alpha-2-delta subunit on voltage-gated calcium channels and reduces calcium influx at nerve terminals, resulting in a decreased release of several neurotransmitters, such as glutamate, norepinephrine, and substance P (Micheva et al., 2003; Staud and Spaeth, 2008). Although the TMS may be used to map cortical function and to measure the neuronal membrane excitability of human cerebral cortex, it does not permit to evaluate changes in cellular neurobiological processes directly (Ziemann et al., 1996a). In healthy subjects, pregabalin does not change the motor thresholds. This indicates that it does not predominantly effect changes of the neuronal membrane excitability (Ziemann et al., 1996b). At present, this disconnection between findings reported in vitro, in contrast with results found in vivo, is an overactivation of mechanisms that reduce excitation and lead to massive releases of GABA from inhibitory interneurons. Thus, the capacity of the GABA-uptake enzymes may be insufficient to remove GABA quickly from the synaptic cleft, thereby favoring postsynaptic GABAB-receptor activation (Di Lazzaro et al., 2004).

Therefore, it is plausible to consider the use of neuronal inhibition indexes to measure the effect of pregabalin on neural substrates, as this effect has been associated with a clinical impact on FM symptoms. Accordingly, the TMS paradigms permit that we evaluate differentially the inhibitory and excitatory neural arrangements, thus, we can measure how pregabalin affects cellular communication. In human studies, it is not possible to identify the direct effects of the drug on specific brain circuits, as it can only be identified on a remote neural network. Physiological measures such as TMS measures, therefore allow a more appropriate qualitative and quantitative evaluation of the effect of drugs in neurobiological systems, than plasma levels or dose rates do (Ziemann, 2004).

Although pregabalin is a drug widely used to treat FM, we have limited evidence that associates its effect in vivo, to the ratio of inhibitory/excitatory at the cortical level in FM. Therefore, to obtain new insights of the effect of pregabalin on the neural imbalance (excitability/inhibition) at the cortical networks in FM, we designed the present cross-over trial. We 
used an experimental paradigm to evaluate if pregabalin could be more effective than a placebo, to improve the neuronal imbalance (excitability/inhibition) in the cortical circuitries in FM patients in contrast to healthy subjects (HS). We compared the acute intragroup effect of pregabalin with the placebo, according to the condition: FM and HS in the current silent period (CSP) and SICI, which are the primary outcomes. The secondary outcomes are the pain pressure thresholds (PPT) and the temperature in ${ }^{\circ} \mathrm{C}$ that evokes a pain score of $6 / 10$ on the numerical pain scale $(0-$ 10) during quantitative sensory testing (QST). We assessed if the basal neuronal plasticity state (i.e., levels of serum neurotrophins BDNF and S100-B protein) could predict the effect of therapeutic approaches on the cortical circuitries.

\section{MATERIALS AND METHODS}

\section{Study Design, Setting, and Participants}

This randomized, double-blind, placebo-controlled crossover study was conducted according to the Declaration of Helsinki in Hospital de Clínicas de Porto Alegre (Rio Grande do Sul, Brazil). The protocol was approved by the IRB (IRB from the Hospital de Clínicas de Porto Alegre - HCPA/Approval number: 14-0624). All volunteers and patients provided written informed consent before participating in this study. Neither the patients nor the pain-free volunteers received monetary or any other compensation for participating in this study. The experimental design, cross-over, assessments, and interventions in each of the two sessions are presented in Figure 1.

\section{Participants}

\section{Pain-Free Control Volunteers}

The volunteers were recruited from the general population by advertisement postings in universities, on the Internet, and in public places in the Porto Alegre area. Subjects were considered eligible to participate if they were female, right-handed, and between 19 and 60 years of age, and were screened for eligibility by phone. They answered a structured questionnaire that assessed the following variables: current acute or chronic pain conditions, use of analgesics in the past week, rheumatologic disease, clinically significant or unstable medical or psychiatric disorders, history of alcohol or substance abuse in the past 6 months, neuropsychiatric comorbidity, and use of psychotropic drugs. Volunteers responding positively to any of these questions and those with contraindications for TMS (Rossi et al., 2009) were excluded. Subjects with Beck depression inventory (BDI) (Warmenhoven et al., 2012) scores higher than 13 were also excluded (Beck et al., 1996).

\section{Fibromyalgia (FM) Subjects}

FM subjects were recruited by directly contacting them from the institutional chronic pain clinic, by referrals from other clinic units, and through media advertising. FM diagnosis adhered to 2010 American College of Rheumatology criteria (Wolfe et al., 2011). All subjects were screened for eligibility by phone. We considered females who reported a pain score on the numerical pain scale (NPS 0-10) greater than 5, in the most days of the last month, were right-handed, and were aged between 19 and 60 years, eligible. The exclusion criteria were previous neurologic diseases, pregnancy, breastfeeding, illicit drugs use, history of alcohol abuse; current use of pregabalin, or failure to respond to pregabalin. We excluded subjects who did not understand Brazilian Portuguese and those with contraindications for TMS according to the guidelines for the use of transcranial magnetic stimulation in clinical practice and research (Rossi et al., 2009). The methods and results sections are reported according to the CONSORT guidelines items. Figure 2 shows the flowchart of the study.

\section{Sample Size}

A superiority test from a crossover design, with a sample size of 16 subjects, divided into two groups with a 1:1: ratio, over two sessions, could test for the intragroup difference of $15 \%$ $(S D=8 \%)$ between the pregabalin or placebo group. The sample size was estimated for both primary outcomes (CPS and SICI) with a variation coefficient of 0.5 , to test inequality and to achieve $90 \%$ power at a $0.1 \%$ significance. To account for the multiple outcomes and potential dropouts, we increased the sample size to 17 .

\section{Interventions}

The intervention involved one oral dose of pregabalin $150 \mathrm{mg}$ acquired from ZODIAC as Prebictal $^{\circledR}$, in solid capsules containing pregabalin $150 \mathrm{mg}$ and excipients. A placebo was manufactured with identical solid capsules containing starch. The capsules were manufactured in such a way that the placebo and active treatment had the same size, color, smell, and flavor. The TMS measures and pain measures were started 90 min after administering pregabalin, since an earlier study had demonstrated that a serum peak of pregabalin occurs at this time in patients when used orally (Bockbrader et al., 2010).

\section{Randomization}

The randomization was generated by computer software by an investigator who was not involved with the assessments. Seventeen subjects with FM were allocated to receive pregabalin or a placebo in the ratio of 1:1. In this incomplete block or crossover trial, each subject received some interventions in two sessions. This means that the allocation in a cross-over manner, in the first and second sessions, was pregabalin $(n=8)$ and placebo $(n=9)$, respectively, and in the second session, pregabalin $(n=9)$ and placebo $(n=8)$, respectively. The allocation of the treatment of HS was performed in a similar manner. The experimental design and interventions in each session are presented in Figure 2.

\section{Blinding}

To control for possible measurement bias, participants were instructed to discuss all aspects related to their treatment only with their treating physician (rather than the research personnel). Before the recruitment phase, opaque envelopes were sealed and numbered sequentially; they contained the treatment allocated. After the subjects agreed to participate in the trial, the envelopes 


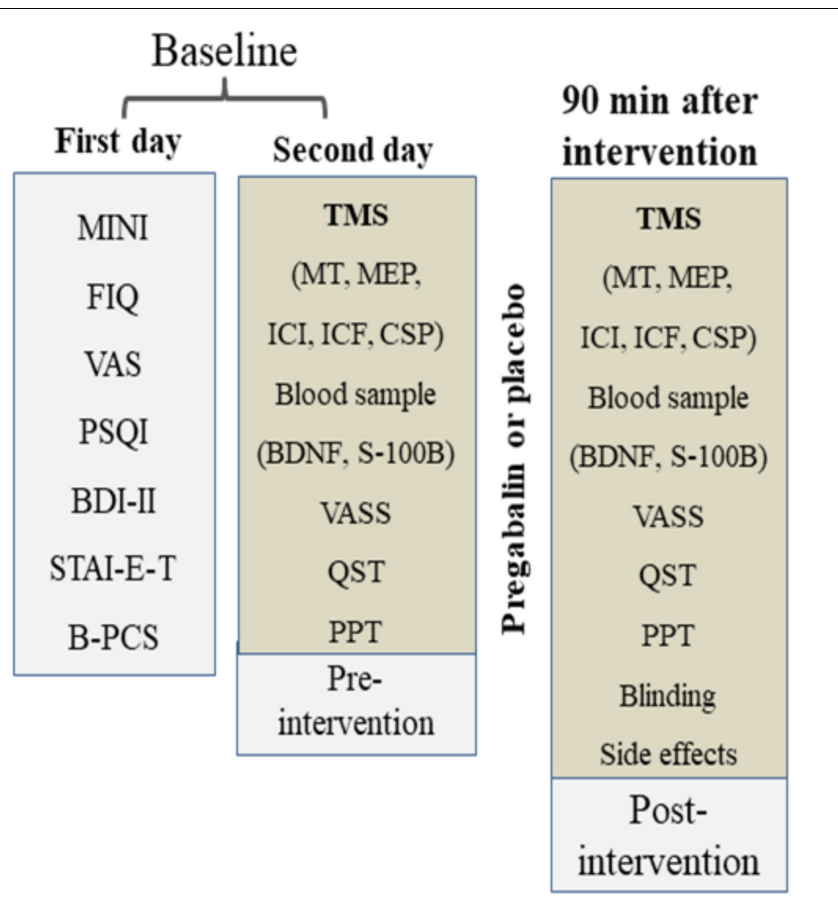

First trail

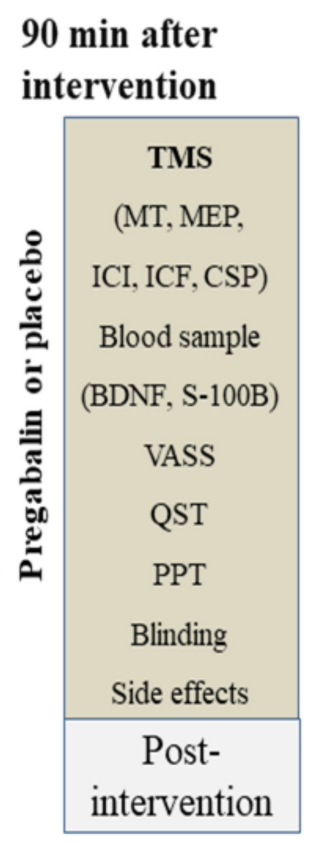

Second trail

FIGURE 1 | Experimental design - cross-over, assessments, and interventions in each one of two sessions. The period between each course was 1 week. Abbreviations: B-PCS, Brazilian Portuguese version of the pain catastrophizing scale; VAS, visual analog scale; STAI-E-T, state-trait anxiety inventory; BDI II, beck depression inventory II; FIQ, fibromyalgia impact questionnaire; PPT, pain pressure threshold; QST, quantitative sensory testing; MINI, mini-international neuropsychiatric interview; VASS, visual analog sleepiness scale; BDNF, brain-derived neurotrophic factor; S100B, S100 calcium-binding protein B; TMS, transcranial magnetic stimulation; TMS measures include motor threshold (MT), motor evoked potential (MEP), short intracortical inhibition (SICI), intracortical facilitation (ICF), and cortical silent period (CSP).

were opened in sequence by a researcher who administered the medications. During the entire protocol timeline, an investigator not involved in the subjects' evaluation was responsible for the blinding and randomization procedure. Other individuals who were involved in the participant's assessments were unaware of the treatment group to which participants belonged. Further, to assess whether blinding was adequate, at the end of the experiment we asked participants to guess whether they had received pregabalin or a placebo and to rate their confidence in the answer on a Likert scale with five categories ("no confidence" to "completely confident").

\section{Outcomes and Measurements}

The primary outcome was the cortical excitability measured by the CSP and the SICI. The secondary outcome was the temperature in the QST that provoked a pain score 6 on the NPS (0-10) and the PPT.

\section{Measurements of Cortical Excitability Using TMS}

The motor cortex excitability was assessed using TMS with a MagPro X100 (MagVenture Company, Farum, Denmark) magnetic stimulator and a figure- 8 coil. The coil was centered over the motor cortex (M1) and held tangentially to the scalp to reach the midline at $45^{\circ}$. To ensure the relaxation of arms and the correct positioning of the hand, subjects were asked to sit in a comfortable reclining chair. Cortical excitability parameters were registered through surface electromyography recordings gathered at the contralateral right first dorsal interosseous muscles using $\mathrm{Ag} / \mathrm{AgCl}$ electrodes. First, the resting motor threshold (RMT) was assessed by obtaining five motor evoked potentials (MEPs) with a peak-to-peak amplitude of $50 \mu \mathrm{V}$ out of 10 consecutive trials. After that, ten MEPs were recorded with an intensity of $130 \%$ of RMT. Moreover, the CSPs were assessed during muscle activity measured on a dynamometer set to approximately $20 \%$ of the maximal force. Accordingly, ten CSPs were recorded using an intensity of $130 \%$ of the RMT. SICI and intracortical facilitation (ICF) were measured using a paired-pulse TMS protocol, and the SICI was assessed using an interstimulus interval (ISI) of 2 and $4 \mathrm{~ms}$; for the ICF measurement, the ISI used was 9 and $12 \mathrm{~ms}$. For ICF and SICI, the conditioning stimulus (first) was set at $80 \%$ of the RMT while the test stimulus (second) was set at $100 \%$ of the individual MEP intensity, and the effect of the conditioning stimulus on the test stimulus was investigated (Kujirai et al., 1993). In total, 30 trials of paired-pulse were conducted in a randomized order (ten for each SICI, ICF, and control stimuli). We included the collection of all amplitudes of the MEPs, SICI, and SICF and the duration of the CSPs in an off-line analysis. SICI was taken as the mean 


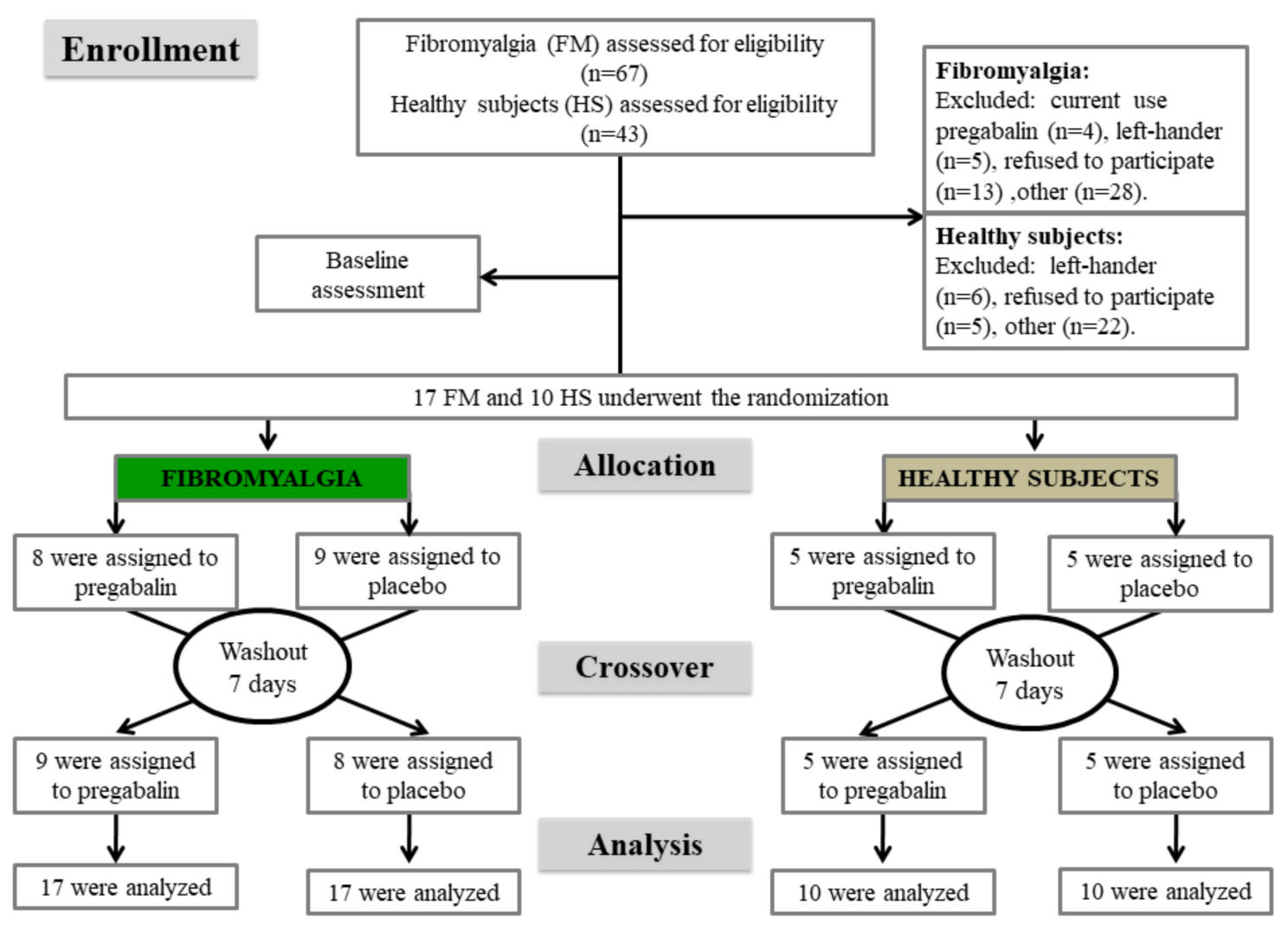

FIGURE 2 | Flowchart showing recruitment and progress through the study.

percentage inhibition at ISIs of 2 and $4 \mathrm{~ms}$, whereas SICF was taken as the mean facilitation at ISIs of 9 and $12 \mathrm{~ms}$. The units for these parameters were: MEP in $\mathrm{mV}$; SICI and ICF in their ratio to the MEP; and the CSP in milliseconds (ms) (Pascual-Leone et al., 1994). Two evaluators with specific training in performing TMS and all cortical excitability measurements preceding the pain assessment, conducted all measurements.

\section{Pain Measurements}

\section{Pain from provocative test}

Quantitative sensory testing was used to provoke a moderate pain $[6 / 10$ on the numerical pain scale (NPS)]. Moderate pain (6/10 NPS) was defined based on thermoalgesic stimuli delivered through a Peltier thermode of a surface of $30 \times 30 \mathrm{~mm}^{2}$ (Schestatsky et al., 2011). The thermode was attached to the skin on the ventral aspect of the mid-forearm, and the temperature was increased at a rate of $1^{\circ} \mathrm{C} / \mathrm{s}$, from $30^{\circ} \mathrm{C}$ to a maximum of $52^{\circ} \mathrm{C}$. The participants were instructed to press a button as soon as they felt moderate pain (6/10) on the NPS ranging from 0 (no pain) to 10 (the worst pain). The temperature necessary to evoke pain (score 6) of each patient was defined as the mean of three assessments performed with an interstimuli interval of 40 s (Schestatsky et al., 2011). A single training session was offered before the assessments to ensure that participants became familiar with the device. The thermode remained on the right ventral forearm, even though it was slightly altered on trials to avoid either response suppression or sensitization of the cutaneous heat nociceptors.

\section{Pressure pain threshold (PPT)}

The PPT was assessed using a digital algometer device (JTECH Medical Industries, Salt Lake City, UT, United States). The algometer's $1 \mathrm{~cm}^{2}$ hard-rubber probe was pressed against the right antecubital fossa with a constantly increasing pressure. The procedure was stopped as soon as the subject indicated uncomfortable pain pressure (when the sensation of pressure changed to one of pain) and the PPT was recorded. This was repeated three times, and the average was calculated and used as the subject's PPT.

\section{Other Instruments and Assessments}

The patients' depressive symptoms were assessed using the Beck depression inventory II (Gomes-Oliveira et al., 2012). To evaluate the sleep quality of patients, the Pittsburgh sleep quality index was used (Bertolazi et al., 2011). The catastrophizing thinking related to pain was evaluated using the Brazilian Portuguese version of the pain catastrophizing scale (B-PCS) (Sehn et al., 2012). Anxiety was evaluated with the refined version of the state-trait anxiety inventory (STAI) (Kaipper et al., 2010). Psychiatric morbidity was defined according to the disorders included in the Mini-international neuropsychiatric interview (MINI) Brazilian version (Amorim, 2000). We used a standardized questionnaire to assess demographic data. An 
independent examiner was trained to administer the pain scales and to conduct the psychological tests. To evaluate the quality of life, we used the fibromyalgia impact questionnaire (FIQ) (Marques et al., 2006). The pain intensity was measured with a $100 \mathrm{~mm}$ visual analog scale (VAS). The VAS scores ranged from no pain (zero) to worst possible pain $(100 \mathrm{~mm})$. Participants were asked to answer the following question using the VAS of pain: ( $i$ ) considering your pain, how intense was your worst pain during the last 24 h? Analgesic use of acetaminophen, non-steroidal antiinflammatory drugs (NSAIDs), or opioid, was defined by the average of analgesics used during the previous week. For data analysis, analgesic use was included as a dichotomous variable (the use of analgesics on less than 4 days per week or use on more than 4 days per week). This approach was chosen because patients' use of chronic pain-rescue analgesics changes each week, depending on their level of pain.

To measure the serum neuroplasticity mediators (BDNF and S100-B protein), we used standard procedures and collected blood at a minimum of $8 \mathrm{~h}$ after fasting early in the morning. All biological materials were collected before applying any intervention (pregabalin or placebo). Plastic tubes were centrifuged for $10 \mathrm{~min}$ at $5,000 \mathrm{~g}$ at $4^{\circ} \mathrm{C}$. Serum was frozen at $-80^{\circ} \mathrm{C}$ until assays were performed. BDNF and S100B serum concentrations were determined using specialized enzymelinked immunosorbent assay (ELISA) kits (BDNF: catalog no. CYT306, the lower detection limit of the kit $=7.8 \mathrm{pg} / \mathrm{mL}$, Chemicon/Millipore, Billerica, MA, United States; S100B: Millipore, MO, United States, catalog no. EZHS100B-33 K, the lower detection limit of the kit $=2.7 \mathrm{pg} / \mathrm{mL}$ ).

\section{Statistical Analysis}

To summarize the main characteristics of the sample, we used traditional descriptive statistics. To compare demographic and clinical measures between conditions (healthy or FM), we used a $t$-test for independent samples to compare continuous variables with parametric distribution and the chi-square or Fisher's exact test for categorical variables. To test for normality, we used the Shapiro-Wilk test. To ensure that the data were normally distributed, we performed a log transformation for the BDNF level.

To examine the changes in the outcome measures across interventions (pregabalin and placebo), we applied a generalized estimating equation (GEE). The GEE analyses were conducted with an exchangeable working correlation structure to account for the correlation between the two sessions from a single participant (Ballinger, 2004). In the GEE model, the sequence of interventions (pregabalin or placebo) serves as the withinsubject variable and accounts for the time variation among repeated measurements present in a longitudinal study design. The factors were the intervention types (pregabalin or placebo) and the conditions (FM patients or HS). In the final models, the interactions among the factors and sequence were also examined. For pairwise comparison of the predicted marginal means, multiple comparison tests were performed for each dependent variable separately. Cramér's V was used as a measure of effect size for chi-square tests.
A MANCOVA model was used to assess the relationship between the BDNF and S100-B, according to the intervention group (pregabalin or placebo) on the SICI and CSP (dependent variables). Considering that pain severity, age, degree of depressive symptoms, analgesic use, and use of psychotropic medications are factors that can affect the biological process of BDNF secretion (Kuczewski et al., 2011; Molendijk et al., 2011), we constructed an adjusted index. A multivariate regression model controlled by multicollinearity was used to obtain an adjusted index used as the surrogate of the BDNF. Statistical significance was stated at a probability under $0.05 P$-value. We used Bonferroni multiple comparison tests to adjust the differences for multiple comparisons. The data were analyzed using SPSS for Windows software version 22.0 (SPSS, Chicago, IL, United States).

\section{RESULTS}

\section{Patient Characteristics}

Seventeen patients with FM and 10 HS were randomized in the study (Figure 2). The clinical and demographic features of patients are shown in Table 1. Regarding the side effects, in the pregabalin group, $85.18 \%(23 / 27)$ of the subjects presented minor side effects (MSE) (mild or moderate nausea, mild or moderate dizziness, mild or moderate dry mouth, mild or moderate headache, mild or moderate drowsiness, and mild xerostomia) and $11.11 \%(3 / 27)$ of subjects presented major side effects (MJSE) (severely blurred vision, severe dizziness, and crippling drowsiness). In the placebo group, $55.55 \%$ (15/27) of the patients presented MSE. The comparisons of the incidence of MSE between the pregabalin and the placebo groups were statistically significant $(P<0.035)$. However, neither the incidence of MSE nor the incidence of MJSE was significant when the groups (FM patients vs. HS) were compared ( $P>0.05$, for all comparisons). Blinding assessments were revealed by three subjects with FM and three HS; $22.2 \%$ $(6 / 27)$ correctly guessed both interventions (pregabalin and placebo). Three reported to be "almost" and "completely" confident, one "moderately" confident, and two "somewhat" or "not confident at all" about the treatment that they had received.

\section{Primary Outcomes: Effects Concerning Cortical Excitability Measured by TMS (CSP and SICI)}

As further analyses were conducted on paired data, means were assessed using the GEE approach and for pairwise comparisons. The between-group changes in cortical excitability measures (CSP and SICI) are shown in Table 2. The post hoc analysis indicated significant differences between the means between pregabalin and the placebo, in the CSP and SICI. However, in HS, these measures did not differ between pregabalin and the placebo (Table 2).

The baseline mean (SD) of the CSP in the FM and healthy groups was 65.79 (19.91) and 67.71 (12.42), respectively 
TABLE 1 | Sample characteristics.

\begin{tabular}{|c|c|c|c|}
\hline Baseline characteristics & FM subjects $n=17$ & Controls $n=10$ & $P$-value \\
\hline Age (years) & $50.5(8.7)$ & $43.7(9.4)$ & 0.07 \\
\hline Body mass index $\left(\mathrm{kg} / \mathrm{m}^{2}\right)$ & $31.3(7.4)$ & $24.2(3.9)$ & 0.00 \\
\hline Education (years) & $10.1(3.8)$ & $17.3(3.4)$ & 0.00 \\
\hline Employed (yes/no) & $10 / 7$ & $10 / 0$ & 0.26 \\
\hline Smoking (yes/no) & $4 / 13$ & $1 / 9$ & 0.62 \\
\hline Pain on the VAS (24 h) (range 0-10) & $7.1(1.8)$ & NA & - \\
\hline Pain on the VAS (last 7 days) (range 0-10) & $7.9(1.9)$ & NA & - \\
\hline Pain duration (months) & $152.47(96.1)$ & NA & - \\
\hline Antidepressants (yes/no) & $14 / 3$ & NA & - \\
\hline Anticonvulsant (yes/no) & $3 / 14$ & NA & - \\
\hline Benzodiazepine (yes/no) & $4 / 13$ & NA & - \\
\hline Analgesic doses (week) & $28.2(22.9)$ & NA & - \\
\hline Beck Depression Inventory II (range 0-63) & $25.4(12.9)$ & $3.8(6.6)$ & 0.00 \\
\hline Brazilian Portuguese Catastrophizing Scale (B-PCS) (range 0-52) & $33.88(12.0)$ & NA & - \\
\hline State anxiety on STAI & $27.3(5.3)$ & $17.9(4.9)$ & 0.00 \\
\hline Trait anxiety on STAI & $29.35(8.1)$ & $16.7(3.4)$ & 0.00 \\
\hline Pittsburgh Sleep Quality Index (range 0-21) & $12.6(4.8)$ & $4.2(2.5)$ & 0.00 \\
\hline FIQ (range 0-100) & $70.39(14.6)$ & NA & - \\
\hline Physically active (yes/no) & $10 / 7$ & $6 / 4$ & 0.95 \\
\hline Sedentary (yes/no) & $7 / 10$ & $4 / 6$ & 0.95 \\
\hline \multicolumn{4}{|c|}{ Psychiatric diagnosis using the Mini International Neuropsychiatric Interview (MINI) } \\
\hline Current depression (yes/no) & $8 / 9$ & NA & - \\
\hline Depression past (yes/no) & $5 / 12$ & NA & - \\
\hline Melancholic depression (yes/no) & $7 / 10$ & NA & - \\
\hline Bipolar I disorder (yes/no) & $3 / 14$ & NA & - \\
\hline Bipolar II disorder (yes/no) & $2 / 15$ & NA & - \\
\hline Generalized anxiety disorder (yes/no) & $5 / 12$ & NA & - \\
\hline Serum BDNF (ng/mL) & $49.8(16.3)$ & $14.8(6.9)$ & 0.00 \\
\hline Serum S100-B (pg/mL) & $18.99(11.52)$ & $25.99(9.12)$ & 0.00 \\
\hline
\end{tabular}

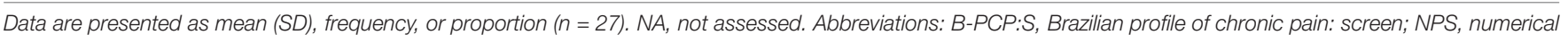

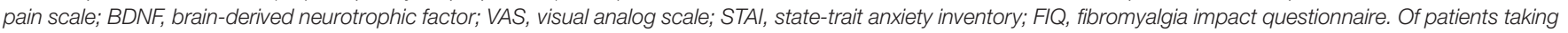
antidepressants, 52.9\% used tricyclic; 47.1\% selective serotonin reuptake inhibitor (SSRI), and 17.6\% serotonin-norepinephrine reuptake inhibitors (SNRIs).

$(\chi 2=0.63, \mathrm{DF}=1 ; P=0.42)$. The mean (SD) in the SICI in FM and HS was $0.37(0.20)$ and $0.29(0.32)$, respectively $(\chi 2=1.24$, $\mathrm{DF}=1 ; P=0.26)$. GEE evaluation revealed that the order in which the intervention - pregabalin or placebo-was administered did not influence their effect either on the CSP $(\chi 2=5.59, \mathrm{DF}=3$; $P=0.13)$ or on the SICI $(\chi 2=3.63, \mathrm{DF}=3 ; P=0.30)$, that is, the first-order carryover effect did not occur.

The GEE model revealed that in FM from before to after intervention, pregabalin increased the CSP by $14 \%$, while the placebo decreased it by $1.58 \%$. It determined an effect size of large magnitude (0.68) (Table 2 ), with a potential clinical impact. In HS, pregabalin and the placebo produced an increase of 12.88 and $14.66 \%$ in the CSP, respectively. The effect was minimal, with no statistical difference or clinical relevance (Cramér's V $=0.19$ ).

Pregabalin decreased SICI from the baseline by $8.10 \%$, while the placebo increased it by $19.56 \%$. Pregabalin produced a substantial size effect (0.54) (Table 2), with a possible relevance in the clinical setting. In $\mathrm{HS}$, pregabalin and the placebo caused an increase of 10.34 and $13.79 \%$ in the SICI, respectively. The effect was minimal, with neither statistical difference nor clinical relevance. The change in means represented as percentages of CPS and SICI are shown in Figures 3A,B.

\section{Secondary Outcomes \\ Pain Measures: Provocative Test Performed to Provoke Pain Score 6 on the NPS (0-10) Using the Quantitative Sensory Testing (QST) and Pressure Threshold (PPT)}

To compare the effect of interventions (pregabalin and placebo) on the means of the temperature that provoked pain score 6 on the NPS (0-10) by the QST as well on the PPT, we used the GEE approach (Table 3). GEE revealed that the order in which the intervention (pregabalin or placebo) was administered did not influence the effect of the intervention on the temperature that provoked pain to score 6 on the NPS $(0-10)$ neither during the QST $(\chi 2=1.71, \mathrm{DF}=3 ; P=0.63)$ nor in the PPT $(\chi 2=0.17$, $\mathrm{DF}=3 ; P=0.68)$. That is, the first-order carryover effect did not occur. Although both the groups (FM patients and HS) were receiving pregabalin, it improves the threshold for the provocative test to produce pain score $6 / 10$ on the NPS with a difference statistically significant only in the FM group. 
TABLE 2 | Primary outcomes.

\begin{tabular}{|c|c|c|c|c|c|c|c|}
\hline & \multicolumn{2}{|c|}{ Mean (SD) } & \multirow[b]{2}{*}{$\%(\mathrm{Cl} 95 \%)^{¥}$} & \multirow[b]{2}{*}{ Wald $\chi^{2}$} & \multirow[b]{2}{*}{ Df } & \multirow[b]{2}{*}{$\boldsymbol{P}$} & \multirow[b]{2}{*}{ Effect size } \\
\hline & Before intervention & After intervention & & & & & \\
\hline \multicolumn{8}{|c|}{ Primary outcomes: cortical excitability measured } \\
\hline \multicolumn{8}{|c|}{ Cortical Salient Period (CSP) } \\
\hline \multicolumn{8}{|c|}{ Fibromyalgia $(n=17)$} \\
\hline Placebo & $65.79(18.91)$ & $64.75(18.08)$ & $-1.58 \%(-57$ to 25.9$)$ & 7.48 & 1 & $0.00^{*}$ & 0.68 \\
\hline Pregabalin & $65.79(18.91)$ & $81.23(15.37)$ & $14 \%$ (4.02 to 21.63$)$ & & & & \\
\hline \multicolumn{8}{|c|}{ Healthy $(n=10)$} \\
\hline Placebo & $64.37(31.35)$ & $75.88(34.52)$ & $17.88 \%(2.22$ to 21.93$)$ & 0.67 & 1 & 0.43 & 0.19 \\
\hline Pregabalin & $64.37(31.35)$ & $77.02(32.24)$ & $16.42 \%$ (3.42 to 25.90$)$ & & & & \\
\hline \multicolumn{8}{|c|}{ Short Intracortical Inhibition (ratio: SICl/test stimulus) } \\
\hline \multicolumn{8}{|c|}{ Fibromyalgia $(n=17)$} \\
\hline Placebo & $0.37(0.20)$ & $0.46(0.18)$ & $19.56 \%(8.10$ to 59.45$)$ & 5.03 & 1 & $0.02^{*}$ & 0.54 \\
\hline Pregabalin & $0.37(0.20)$ & $0.34(0.20)$ & $-8.82 \%(-26$ to 46.00$)$ & & & & \\
\hline \multicolumn{8}{|c|}{ Healthy $(n=10)$} \\
\hline Placebo & $0.29(0.32)$ & $0.33(0.12)$ & $12.12 \%(-24.00$ to 41.00$)$ & 0.98 & 1 & 0.32 & 0.18 \\
\hline Pregabalin & $0.29(0.32)$ & $0.31(0.16)$ & $6.45 \%(-23.00$ to 38.00$)$ & & & & \\
\hline
\end{tabular}

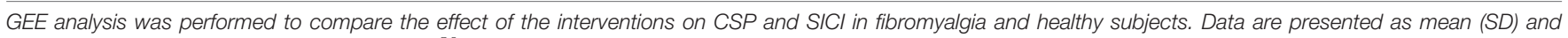

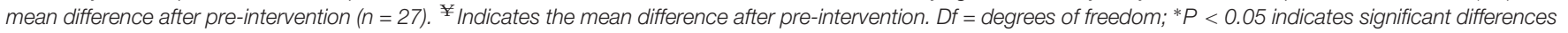

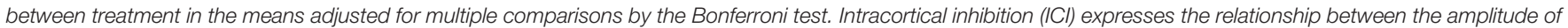

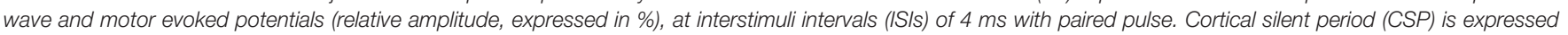

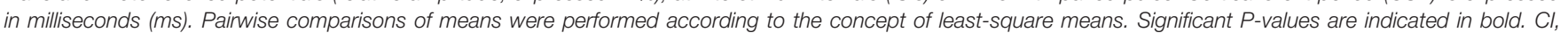

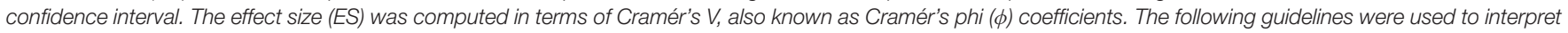
the magnitude of the ES: small, 0.1 to 0.3; medium, 0.30 to 0.50; and large, 0.50 or higher. Values to be considered with clinical relevance should be a large ES.

Pregabalin increased the threshold for the provocative test to produce a pain score of $6 / 10$ on the NPS from before to after intervention by $2.67 \%$ in the FM group. It was observed that the provocative test performed to produce a pain score of $6 / 10$ on the NPS (0-10) resulted in an effect size with a large magnitude (Cramér's $V=1.05$ ) that is compatible with decreased pain perception (Table 3 ).

Pregabalin increased the PPT from before to after intervention by $9.96 \%$. The size effect of pregabalin on the PPT compared to the placebo presents a large effect size (0.57) (Table 3), and in HS, the effect of pregabalin compared to that of the placebo produced a tiny size effect (0.08) (Table 3).

\section{Secondary Analysis: Relationship Between Serum Markers of Neuroplasticity Protein S100B and BDNF on the Effect of Treatment on Cortical Inhibition (CSP and SICI)}

As presented in Table 1, serum BDNF and S100-B protein differ between healthy volunteers and FM patients. We observed that serum BDNF and S100-B in those with FM had significantly higher levels of both biomarkers in comparison to that in HS. To explore the effect of the intervention in cortical excitability to account for the neuroplasticity state before the intervention, we conducted an exploratory secondary analysis as presented below. First, considering the variables that comprise the confounding factor to be associated with either BDNF secretion and cortical excitability, we constructed an adjusted the index of BDNF, using a multivariate regression model to account for the influence of pain severity, age, depressive symptoms, analgesic use, and use of psychotropic medications. After the adjustment, the covariate retained in the model was the analgesic doses used weekly ( $r$-squared $=0.24$, standard $\beta$ coefficient $=-0.42, t=-3.36, P<0.01$ ). After constructing this BDNF-adjusted index, we ran a multivariate linear regression model with SICI and CSP as dependent variables, the BDNF-adjusted index, the serum S100-B protein, and their own interaction with treatment (pregabalin or placebo) as independent variables. The model is presented in Table 4. This analysis showed a significant relationship between the level of these markers and the CSP (Wilks' $\lambda=0.37, F=20.49$, $P<0.001)$.

The multiple regression analysis showed that the CSP, after the intervention, is correlated with serum levels of markers before the intervention. It was positively associated with the BDNF-adjusted index and negatively correlated with serum S100-B (Table 4). We did not observe an interaction between interventions (pregabalin or placebo) and the BDNF or $\mathrm{S} 100-\mathrm{B}(P>0.05)$. This preliminary result suggests that BDNF and S100-B protein are correlated with the CSP, but these neurotrophic factors do not influence the effect of interventions (pregabalin or placebo) in SICI.

\section{DISCUSSION}

Our findings attempt to define the neural mechanisms at corticomotor pathways as a novel avenue to understanding the mechanisms involved in the central disinhibition in FM, as indicated by the effect of pregabalin on CSP and SICI. 
A

Fibromyalgia

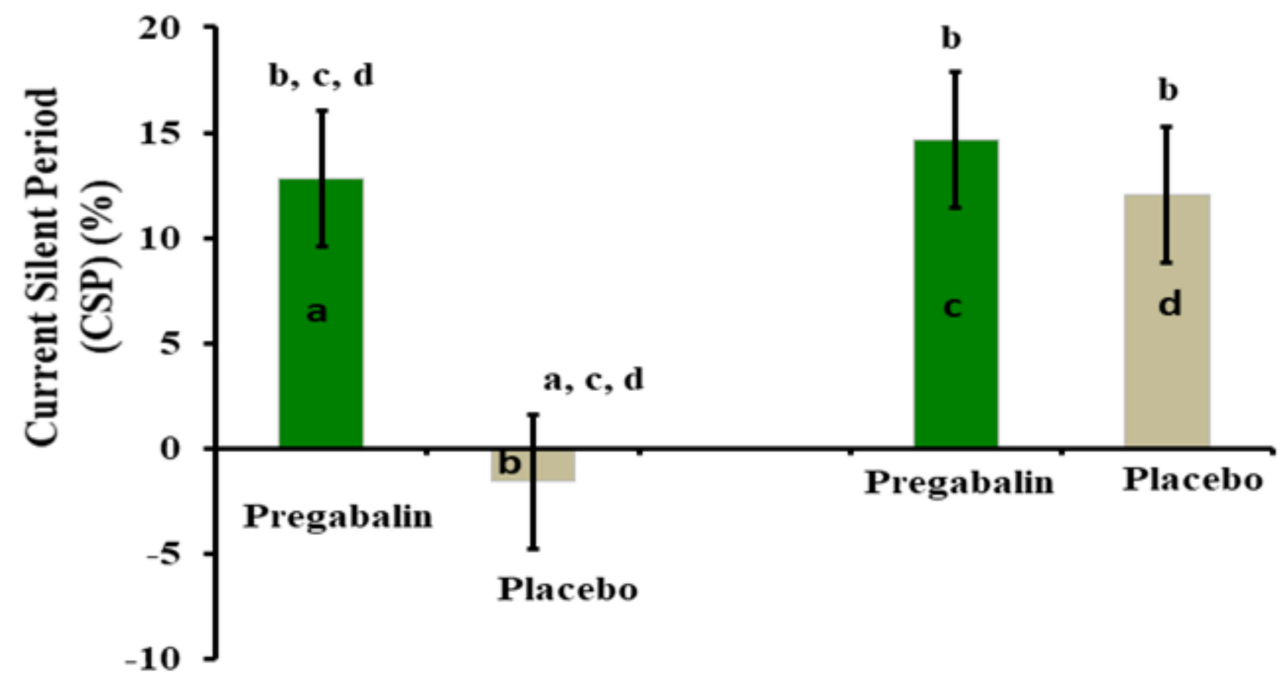

B

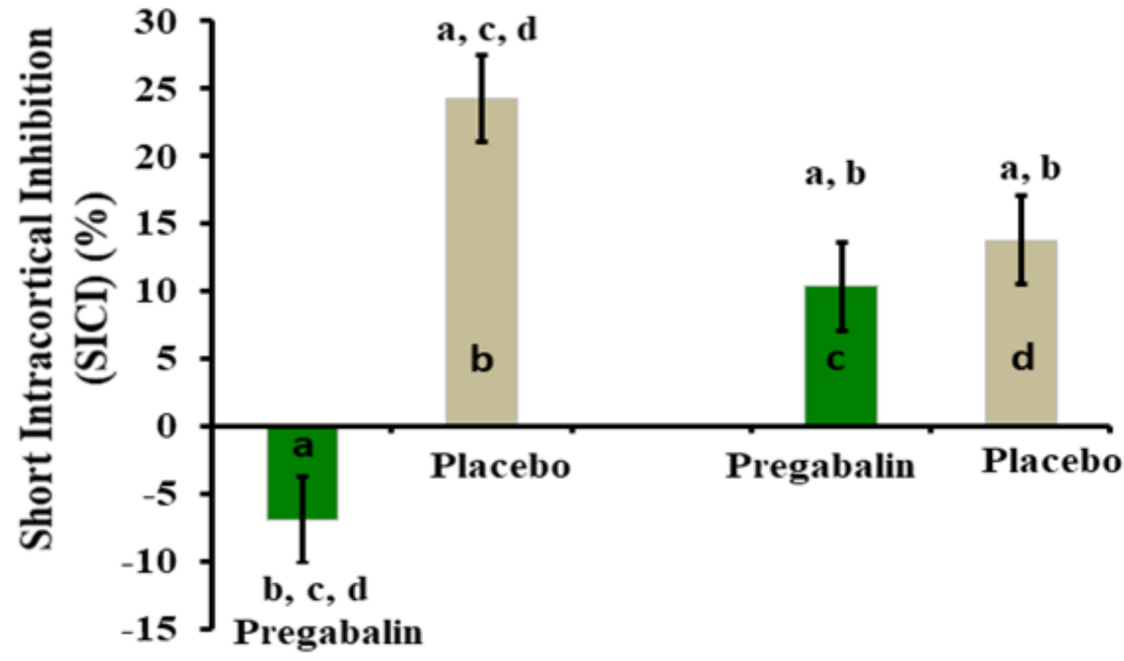

FIGURE 3 | (A,B) Percentage of change from pre- to post-intervention. Bars indicate the mean and the standard error of the mean (SEM). The groups are identified by letters: FM group treated with pregabalin (a) and placebo (b). HS group treated with pregabalin (c) and placebo (d). All comparisons were performed by using a GEE model, followed by the Bonferroni correction for post hoc multiple comparisons. Post hoc differences between groups are indicated via superscript letters.

Additionally, in FM, the effects of pregabalin enhanced the PPT, and it improved pain perception. These results suggest that an interaction occurred between the disinhibition at motor cortex and the modulation of pain in FM and its behavioral correlates. In addition, they show that the baseline serum levels of neurotrophic factors as BDNF and S-100B protein might influence different responses on the corticomotor excitability as demonstrated by their correlations with the CSP.
The acute effect of pregabalin on cortical excitability parameters (i.e., CSP and SICI) and pain measures was distinct in FM patients compared to HS. In FM patients, the pronounced effect of pregabalin in the inhibitory cortical circuits as measured by CSP highlight its impact on motor cortical inhibition. However, in HS, neither the placebo nor pregabalin increased cortical inhibition (Figure 3A). These results support the concept that pregabalin is more effective in modulating nociceptive 
TABLE 3 | Secondary outcomes.

\begin{tabular}{|c|c|c|c|c|c|c|c|}
\hline & \multicolumn{2}{|c|}{ Mean (SD) } & \multirow[b]{2}{*}{ Cl $95 \%$} & \multirow[b]{2}{*}{ Wald $\chi^{2}$} & \multirow[b]{2}{*}{ Df } & \multirow[b]{2}{*}{$\boldsymbol{P}$} & \multirow[b]{2}{*}{ Effect size } \\
\hline & Before intervention & After intervention & & & & & \\
\hline \multicolumn{8}{|c|}{ Secondary outcomes - treatment effect on pain outcomes } \\
\hline \multicolumn{8}{|c|}{ Secondary outcomes: pain measures } \\
\hline \multicolumn{8}{|c|}{ Provocative test to induce pain score 6 on the NPS $(0-10)$ using the QST (temperature ${ }^{\circ} \mathrm{C}$ ) } \\
\hline \multicolumn{8}{|c|}{ Fibromyalgia $(n=17)$} \\
\hline Placebo & $42.22(4.07)$ & $40.27(4.07)$ & (38.31 to 42.23$)$ & 18.0 & 1 & $0.00^{*}$ & 1.05 \\
\hline Pregabalin & $42.22(4.07)$ & $43.39(4.36)$ & (41.10 to 45.28$)$ & & & & \\
\hline \multicolumn{8}{|c|}{ Healthy $(n=10)$} \\
\hline Placebo & $44.55(5.52)$ & $44.61(3.58)$ & (42.89 to 46.33$)$ & 1.18 & 1 & 0.28 & NE \\
\hline Pregabalin & $44.55(5.52)$ & $45.09(3.62)$ & (43.35 to 46.83$)$ & & & & \\
\hline \multicolumn{8}{|c|}{ Pain pressure threshold $\left(\mathrm{kg} / \mathrm{cm}^{2}\right)$} \\
\hline \multicolumn{8}{|c|}{ Fibromyalgia $(n=17)$} \\
\hline Placebo & $2.44(1.34)$ & $2.30(1.03)$ & (1.80 to 2.80$)$ & 5.46 & 1 & $0.01^{*}$ & 0.57 \\
\hline Pregabalin & $2.44(1.34)$ & $2.71(0.90)$ & (2.12 to 3.07$)$ & & & & \\
\hline \multicolumn{8}{|c|}{ Healthy $(n=10)$} \\
\hline Placebo & $4.32(1.03)$ & $4.65(2.63)$ & (3.38 to 5.92$)$ & 0.06 & 1 & 0.80 & NE \\
\hline Pregabalin & 4.32 (1.03) & 4.62 (2.39) & (3.47 to 5.78 ) & & & & \\
\hline
\end{tabular}

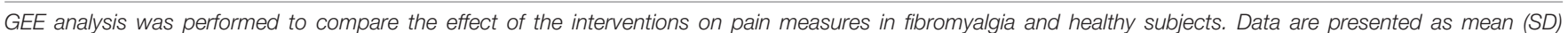

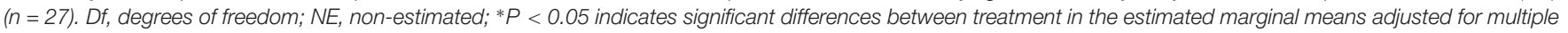
comparisons by the Bonferroni test.

TABLE 4 | Multiple regression models of the relationship between the effect of serum levels of S-100 protein and BDNF on the effect of interventions on the SICI and $\operatorname{CSP}(n=17)$.

\begin{tabular}{|c|c|c|c|c|c|c|}
\hline Dependent variable & Type III sum of squares & $d f$ & Mean square & $\boldsymbol{F}$ & $\boldsymbol{P}$ & Partial eta squared \\
\hline Short intracortical inhibition (SICl) & 0.46 & 4 & 0.12 & 1.77 & 0.17 & 0.22 \\
\hline \multirow[t]{2}{*}{ Cortical silent period (CSP) } & 3881.54 & 4 & 970.38 & 4.77 & 0.005 & 0.43 \\
\hline & $\mathrm{B}$ & Std. error & $t$ & $P$ & Cl $95 \%$ & \\
\hline \multicolumn{7}{|c|}{ Dependent variable: short intracortical inhibition (SICI) } \\
\hline Intercept & 0.75 & 0.22 & 3.32 & 0.00 & (0.29 to & \\
\hline Serum S100-B protein & 0.01 & 0.022 & 0.63 & 0.53 & $(-0.03 t$ & \\
\hline BDNF adjusted index & -0.02 & 0.011 & -1.78 & 0.09 & $(-0.04 t$ & 03) \\
\hline \multicolumn{7}{|l|}{ Interaction } \\
\hline Serum S100-B protein* treatment & -0.05 & 0.012 & -0.36 & 0.71 & $(-0.06 t$ & \\
\hline BDNF adjusted index * treatment & 0.06 & 0.006 & 1.01 & 0.32 & $(-0.08 t$ & \\
\hline \multicolumn{7}{|c|}{ Dependent variable: cortical silent period (CSP) } \\
\hline Intercept & 62.12 & 12.61 & 4.93 & 0.00 & $(36.15 \mathrm{t}$ & 09) \\
\hline Serum S100-B protein & -2.68 & 1.24 & -2.16 & 0.04 & $(-5.24 t$ & $.12)^{* *}$ \\
\hline BDNF adjusted index & 1.41 & 0.601 & 2.33 & 0.02 & (0.16 to & \\
\hline \multicolumn{7}{|l|}{ Interaction } \\
\hline Serum S100-B protein* treatment & 0.84 & 0.69 & 1.22 & 0.23 & $(-0.57 t$ & \\
\hline BDNF adjusted index * treatment & -0.43 & 0.31 & -1.38 & 0.18 & $(-1.08 t$ & \\
\hline
\end{tabular}

**Interaction between dependent variable (S100B or BDNF)*trearment (factor).

transmission when the neural excitability in the nociceptive input is enhanced. Similarly, the analgesic and anxiolytic effects of pregabalin occur particularly under conditions of hyperexcitability (i.e., pain and anxiety) to reduce the release of excitatory neurotransmitters and peptide neuromodulators (Tanga et al., 2006), leading to an overbalance of inhibition with proportional releases of GABA from the inhibitory interneurons.
The increase in the duration in CSP in FM patients involves the GABAB receptors (Siebner et al., 1998; Werhahn et al., 1999) and can reveal the extent of deactivated areas by neuronal hyperexcitability. This hypothesis is plausible according to studies with a cognitive task of increasing difficulty, as well as experimental pain studies that assess the change perception of pain when the resource attentional shift toward regions activated (Coghill et al., 1999). In addition, a previous 
study with FM patients showed that pregabalin reduced the signal evoked in functional magnetic resonance imaging in areas involved in pain processing (i.e., insula, thalamus, and precuneus) (Kim et al., 2013). In the same way, two other findings suggest the effect of pregabalin in pain processing: first, the absence of a similar modulation during the placebo use in FM patients, and second, the lack of pregabalininduced changes on CSP in HS. Therefore, the effect of pregabalin on pain measures can be interpreted in the sense that, the disinhibition state involves multiple inhibitory mechanisms, which can change the sensory signals to the pain matrix. This result suggests that its effects enhance the balance of the excitatory/inhibitory systems in the corticomotor area.

The reduction in SICI induced by pregabalin indicates a mediated effect by GABAA receptors. It is important to note that this effect of pregabalin in SICI was observed only in the FM, and we did not find a significant effect of pregabalin on the cortical excitability in pain measures in HS. However, in a previous study, a similar result was found in HS with a dosage of $300 \mathrm{mg}$ (Lang et al., 2006). The disagreement, related to pregabalin's effect on HS, may be explained by the difference in the dosage. Our results may be of clinical relevance because they can help create a roadmap for customizing treatment in FM, based on an individual's characteristics. The results are relevant to investigate novel therapeutic approaches that target the motor cortex (i.e., transcranial direct current stimulations tDCS and TMS), which appear to be a key system for the endogenous modulation of pain. Accordingly, the results suggest that M1 may be an entry port to assess the complex pain-related neural network, as well as to understand the role of M1 to inhibit or interrupt pain signals. Furthermore, they strengthen the notion that the use of neurophysiological measures in combination with neuropharmacological challenges provide an ideal opportunity to determine the dose, to produce a specific effect on the distinct brain systems involved in pain processing.

We observed that the placebo effect increased the SICI in FM, suggesting that expectancy raises corticospinal excitability. Accordingly, previous studies showed that the expectancyinduced increases in the MEP amplitude, that could result from the decreased excitability of intracortical inhibitory networks, or the increased excitability of intracortical facilitatory systems (van Elswijk et al., 2007). Another study on pain has shown that verbal instructions to participants, to focus on one body part, enhanced the placebo response (Geers et al., 2006). This data set suggests that different verbal information can direct attention toward or away from the body which can impact the response to the placebo. The assumption here is that the expectation induced through the placebo procedure can influence cognitive processing (Geers et al., 2006). However, we realize that we did not find this effect in the HS placebo group. We do not know any apparent reason to explain this finding; it is possible that a stimulus related to pain or treatment promotes selective attentional activation of neural networks and this justifies the higher cortical excitability observed in the FM placebo group.
As previously mentioned, the excitation of the primary motor cortex's (M1) pyramidal neurons, is a target to a goal-setting process used to determine the intervention outcomes using a non-invasive method, such as magnetic pulses, to stimulate a restricted part of the cortex. In this context, the effect of pregabalin might help characterize the functional dysconnectivity of cortical networks that have been identified as important to assess specific aspects of the motor cortex function in pain physiopathology. At the same time, the effect of pregabalin in CSP and SICI is translated onto the intracortical circuitry within the motor cortex. This effect of pregabalin within M1 suggests that pregabalin modulates the corticospinal output. The thalamus is an important structure that mediates different components of pain and is also involved in the descending inhibition to modulate nociceptive inputs at the dorsal horn of the spinal cord. Thus, this sum of findings supports the hypothesis that the effect of pregabalin on pain and in cortical inhibition occurs by its modulatory force, in the thalamocortical connections projected to the primary somatosensory cortex. These results are in line with the notion that, inhibition is physiologically separate from excitation and has a lower threshold than that of excitation.

The correlation between CSP with BDNF and S-100B protein provide some evidence that these neuroplasticity factors might show at some level, the changes in the excitatory/inhibitory balance in the CNS involved in the transmission of nociceptive inputs. The BDNF is positively correlated to CSP; this suggests that the BDNF is involved in motor cortex disinhibition. This finding agrees with a recent study which found a positive correlation between SICI and the BDNF. It suggests that an interaction of this neurotrophic factor with the disinhibition of the motor cortex exists (Caumo et al., 2016). This result can reflect the BDNF influence on the GABAA receptors modulation (Ziemann et al., 2015). On the other hand, the correlation of the BDNF with the CSP also suggests its relationship to the GABAB receptor. According to experimental evidence, the $G A B A B$ receptor activation triggers the BDNF release and promotes the functional maturation of GABAergic synapses that increases the level of GABAA receptors at the plasma membrane (Kuczewski et al., 2011). The negative correlation of the S-100B protein with the CSP suggests that it is allied with the motor cortex excitability. Although the exact mechanism underlying this association is unclear, this is a plausible hypothesis because, the S-100B protein can increase intracellular free calcium concentrations to regulate neuron excitability (Selinfreund et al., 1991; Barger and Van Eldik, 1992). S-100B is the hallmark of astrocytic activation, and it might stimulate the astrocyte proliferation in vitro (Selinfreund et al., 1991; Barger and Van Eldik, 1992); in addition, experimental studies have demonstrated that GABAB receptors are expressed on cultured astrocytes (Selinfreund et al., 1991). Additionally, an experimental study on neuropathic pain showed that it is related to allodynia (Tanga et al., 2006). However, in clinical studies, we cannot isolate the effect of each system; for that reason, we cannot affirm that these are of the cause-consequence relationship type. 
Several issues concerning the design of our study must be addressed. First, the absence of first-order carryover effects showed that the sequence in which participants received the intervention was not aliased with treatment differences. The crossover design permits to increase the uniformity, and it eliminates the between-subject variability because, the interventions under investigation were evaluated within the same subject (Grizzle, 1965). Given that participants act as their controls, the analyses could be based on paired data (using paired tests) (Brown, 1980; Maclure, 1991). Second, the sedative effect of pregabalin and its side effects can affect the blinding. The sleepiness might affect mainly the pain measures; however, the sleepiness score was included in the model and its influence did not statistically affect the outcomes related to pain. Third, the rate of guessing about the intervention (pregabalin or placebo) was similar between healthy and FM subjects. Additionally, our objective surrogates were less prone to bias, i.e., cortical excitability measures and pain provocation tests, and it was unlikely that un-blinding would change our conclusions. Fourth, we included only females because FM is more prevalent in women. Women are prone to activation upon negative emotional responses (i.e., stress, fear, and anxiety) and a higher anxiety trait has been associated with an imbalance in the excitability of the corticospinal tract (Vidor et al., 2014). Fifth, although a limitation of the current study was that we used the single baseline, and as psychophysical parameters are subject to a sizeable intersession variability, the post-treatment changes would be more comparable with the within-session baseline. Hence, this is a limitation that must be taken into account in the interpretation of our findings. However, we chose a cross-over design in which we had a single-subject design, to permit that the subjects serve as their control. This way, it is possible that this factor could have a lesser impact on our results. Finally, although the present findings are important to understand the possible neurobiological mechanisms of central excitatory/inhibitory balance systems in fibromyalgia, they do not support therapeutic decision-making in clinical settings.

These results suggest that pregabalin's effect on cortical neural networks occurs, particularly under basal neuronal hyperexcitability, because its impact on the cortical excitability and in pain measures was observed only in the FM group. They suggest that pregabalin can reduce the inhibitory connections in specific neural networks, while it can increase the excitatory activity in others. However, they indicate that pregabalin effect is dependent on the baseline neuroplasticity state.

\section{REFERENCES}

Amorim, P. (2000). Mini International Neuropsychiatric Interview (MINI): validação de entrevista breve para diagnóstico de transtornos mentais. Rev. Bras. Psiquiatr. 22, 106-115. doi: 10.1590/S1516-444620000003 00003

Ballinger, G. A. (2004). Using generalized estimating equations for longitudinal data analysis. Organ. Res. Methods 7, 127-150. doi: 10.1177/1094428104263672

Barger, S. W., and Van Eldik, L. J. (1992). S100 beta stimulates calcium fluxes in glial and neuronal cells. J. Biol. Chem. 267, 9689-9694.

\section{ETHICS STATEMENT}

This study was carried out in accordance with the principles of the Declaration of Helsinki and recommendations of the Ethics and Research Committee of Hospital de Clínicas de Porto Alegre, according to Brazilian Laws for Clinical Research in Humans. The protocol was approved by the Ethics and Research Committee of Hospital de Clínicas de Porto Alegre.

\section{AUTHOR CONTRIBUTIONS}

AD conceived the study, participated in its design and coordination, participated in the sequence alignment, and drafted the manuscript. MS participated in the design of the study, performed the statistical analysis, and drafted the manuscript. JD-S participated in the sequence alignment and drafted the manuscript. AS participated in the sequence alignment. IST participated in the design of the study and performed the statistical analysis. FF participated in the study design and coordination and helped to draft the manuscript. WC conceived the study, participated in its design, sequence alignment and coordination, and helped to draft the manuscript.

\section{FUNDING}

This research was supported by grants and material support from the following Brazilian agencies: Committee for the Development of Higher Education Personnel - CAPES PNPD/CAPES (Grants to AD and JD-S) (material support). National Council for Scientific and Technological Development CNPq (Grants to IST and WC). Postgraduate Program in Medical Sciences at the School of Medicine of the Federal University of Rio Grande do Sul (material support). International Cooperation Program - CAPES (023/11) (WC and FF). Postgraduate Research Group at the Hospital de Clínicas de Porto Alegre - FIPE HCPA (material support). Foundation for Support of Research at Rio Grande do Sul (FAPERGS) (material support). Brazilian Innovation Agency (FINEP) process number - 1245/13 (IST and WC). Research grant: National Council for Scientific and Technological Development-CNPq (IST 302345/2011-6 and WC WC301256/2013-6).

Beck, A. T., Steer, R. A., Ball, R., and Ranieri, W. (1996). Comparison of beck depression inventories -IA and -II in psychiatric outpatients. J. Pers. Assess. 67, 588-597. doi: 10.1207/s15327752jpa6703_13

Bertolazi, A. N., Fagondes, S. C., Hoff, L. S., Dartora, E. G., da Silva Miozzo, I. C., de Barba, M. E. F., et al. (2011). Validation of the Brazilian Portuguese version of the Pittsburgh sleep quality index. Sleep Med. 12, 70-75. doi: 10.1016/j.sleep. 2010.04.020

Bockbrader, H. N., Radulovic, L. L., Posvar, E. L., Strand, J. C., Alvey, C. W., Busch, J. A., et al. (2010). Clinical pharmacokinetics of pregabalin in healthy volunteers. J. Clin. Pharmacol. 50, 941-950. doi: 10.1177/0091270009352087 
Brown, B. W. (1980). The crossover experiment for clinical trials. Biometrics 36, 69-79. doi: $10.2307 / 2530496$

Caipa, A., Alomar, M., and Bashir, S. (2018). TMS as tool to investigate the effect of pharmacological medications on cortical plasticity. Eur. Rev. Med. Pharmacol. Sci. 22, 844-852. doi: 10.26355/EURREV_201802_14321

Caumo, W., Deitos, A., Carvalho, S., Leite, J., Carvalho, F., Dussán-Sarria, J. A., et al. (2016). Motor cortex excitability and BDNF levels in chronic musculoskeletal pain according to structural pathology. Front. Hum. Neurosci. 10:357. doi: $10.3389 /$ fnhum.2016.00357

Coghill, R. C., Sang, C. N., Maisog, J. M., and Iadarola, M. J. (1999). Pain intensity processing within the human brain: a bilateral, distributed mechanism. J. Neurophysiol. 82, 1934-1943. doi: 10.1152/jn.1999.82.4.1934

Di Lazzaro, V., Oliviero, A., Pilato, F., Saturno, E., Dileone, M., Mazzone, P., et al. (2004). The physiological basis of transcranial motor cortex stimulation in conscious humans. Clin. Neurophysiol. 115, 255-266. doi: 10.1016/j.clinph. 2003.10.009

Di Lazzaro, V., Pilato, F., Dileone, M., Ranieri, F., Ricci, V., Profice, P., et al. (2006). GABAA receptor subtype specific enhancement of inhibition in human motor cortex. J. Physiol. 575(Pt 3), 721-726. doi: 10.1113/jphysiol.2006.114694

Galhardoni, R., Correia, G. S., Araujo, H., Yeng, L. T., Fernandes, D. T., Kaziyama, H. H., et al. (2015). Repetitive transcranial magnetic stimulation in chronic pain: a review of the literature. Arch. Phys. Med. Rehabil. 96, S156-S172. doi: 10.1016/j.apmr.2014.11.010

Geers, A. L., Helfer, S. G., Weiland, P. E., and Kosbab, K. (2006). Expectations and placebo response: a laboratory investigation into the role of somatic focus. J. Behav. Med. 29, 171-178. doi: 10.1007/s10865-005-9040-5

Gomes-Oliveira, M. H., Gorenstein, C., Neto, F. L., Andrade, L. H., and Wang, Y. P. (2012). Validation of the Brazilian Portuguese Version of the beck depression inventory-II in a community sample. Rev. Bras. Psiquiatr. 34, 389-394. doi: 10.1016/j.rbp.2012.03.005

Grizzle, J. E. (1965). The two-period change-over design and its use in clinical trials. Biometrics 21, 467-480. doi: 10.2307/2528104

Kaipper, M. B., Chachamovich, E., Hidalgo, M. P. L., da Silva Torres, I. L., and Caumo, W. (2010). Evaluation of the structure of Brazilian State-Trait Anxiety Inventory using a Rasch psychometric approach. J. Psychosom. Res. 68, 223-233. doi: 10.1016/j.jpsychores.2009.09.013

Kim, S.-H., Lee, Y., Lee, S., and Mun, C.-W. (2013). Evaluation of the effectiveness of pregabalin in alleviating pain associated with fibromyalgia: using functional magnetic resonance imaging study. PLoS One 8:e74099. doi: 10.1371/journal. pone.0074099

Kuczewski, N., Fuchs, C., Ferrand, N., Jovanovic, J. N., Gaiarsa, J.-L. L., and Porcher, C. (2011). Mechanism of GABAB receptor-induced BDNF secretion and promotion of GABAA receptor membrane expression. J. Neurochem. 118, 533-545. doi: 10.1111/j.1471-4159.2011.07192.x

Kujirai, T., Caramia, M. D., Rothwell, J. C., Day, B. L., Thompson, P. D., Ferbert, A., et al. (1993). Corticocortical inhibition in human motor cortex. J. Physiol. 471, 501-519. doi: 10.1113/jphysiol.1993.sp019912

Lang, N., Sueske, E., Hasan, A., Paulus, W., and Tergau, F. (2006). Pregabalin exerts oppositional effects on different inhibitory circuits in human motor cortex: a double-blind, placebo-controlled transcranial magnetic stimulation study. Epilepsia 47, 813-819. doi: 10.1111/j.1528-1167.2006.0 0544.x

Latremoliere, A., and Woolf, C. J. (2009). Central sensitization: a generator of pain hypersensitivity by central neural plasticity. J. Pain 10, 895-926. doi: 10.1016/j. jpain.2009.06.012

Maclure, M. (1991). The case-crossover design: a method for studying transient effects on the risk of acute events. Am. J. Epidemiol. 133, 144-153. doi: 10.1093/ oxfordjournals.aje.a115853

Marques, A. P., Santos, A. M. B., Matsutani, L. A., and Lage, L. V. (2006). Validation of the Brazilian version of the Fibromyalgia Impact Questionnaire (FIQ). Rev. Bras. Reumatol. 46, 24-31.

McDonnell, M. N., Orekhov, Y., and Ziemann, U. (2006). The role of GABAB receptors in intracortical inhibition in the human motor cortex. Exp. Brain Res. 173, 86-93. doi: 10.1007/s00221-006-0365-2

Mease, P. J., Clauw, D. J., Arnold, L. M., Goldenberg, D. L., Witter, J., Williams, D. A., et al. (2005). Fibromyalgia syndrome. J. Rheumatol. 32, 2270-2277.
Micheva, K. D., Buchanan, J., Holz, R. W., and Smith, S. J. (2003). Retrograde regulation of synaptic vesicle endocytosis and recycling. Nat. Neurosci. 6, 925-932. doi: 10.1038/nn1114

Molendijk, M. L., Bus, B. A., Spinhoven, P., Penninx, B. W., Kenis, G., Prickaerts, J., et al. (2011). Serum levels of brain-derived neurotrophic factor in major depressive disorder: state-trait issues, clinical features and pharmacological treatment. Mol. Psychiatry 16, 1088-1095. doi: 10.1038/mp.2010.98

Pascual-Leone, A., Valls-Solé, J., Wassermann, E. M., and Hallett, M. (1994). Responses to rapid-rate transcranial magnetic stimulation of the human motor cortex. Brain 117, 847-858. doi: 10.1093/brain/117.4.847

Rossi, S., Hallett, M., Rossini, P. M., Pascual-Leone, A., and Safety of Tms Consensus Group. (2009). Safety, ethical considerations, and application guidelines for the use of transcranial magnetic stimulation in clinical practice and research. Clin. Neurophysiol. 120, 2008-2039. doi: 10.1016/j.clinph.2009. 08.016

Salerno, A., Thomas, E., Olive, P., Blotman, F., Picot, M. C., and Georgesco, M. (2000). Motor cortical dysfunction disclosed by single and double magnetic stimulation in patients with fibromyalgia. Clin. Neurophysiol. 111, 994-1001. doi: 10.1016/S1388-2457(00)00267-4

Schestatsky, P., Stefani, L. C., Sanches, P. R., Silva Junior, D. P., da Torres, I. L. S., Dall-Agnol, L., et al. (2011). Validation of a Brazilian quantitative sensory testing (QST) device for the diagnosis of small fiber neuropathies. Arq. Neuro Psiquiatr. 69, 943-948. doi: 10.1590/S0004-282X2011000700019

Sehn, F., Chachamovich, E., Vidor, L. P., Dall-Agnol, L., de Souza, I. C., Torres, I. L., et al. (2012). Cross-cultural adaptation and validation of the brazilian portuguese version of the pain catastrophizing scale. Pain Med. 13, 1425-1435. doi: $10.1111 / j .1526-4637.2012 .01492 . x$

Selinfreund, R. H., Barger, S. W., Pledger, W. J., and Van Eldik, L. J. (1991). Neurotrophic protein S100 beta stimulates glial cell proliferation. Proc. Natl. Acad. Sci. U.S.A. 88, 3554-3558. doi: 10.1073/pnas.88.9.3554

Siebner, H. R., Dressnandt, J., Auer, C., and Conrad, B. (1998). Continuous intrathecal baclofen infusions induced a marked increase of the transcranially evoked silent period in a patient with generalized dystonia. Muscle Nerve 21, 1209-1212. doi: 10.1002/(SICI)1097-4598(199809)21:9<1209::AID-MUS15>3. $0 . \mathrm{CO} ; 2-\mathrm{M}$

Staud, R., and Spaeth, M. (2008). Psychophysical and neurochemical abnormalities of pain processing in fibromyalgia. CNS Spectr. 13, 12-17. doi: 10.1017/ S109285290002678X

Tanga, F. Y., Raghavendra, V., Nutile-McMenemy, N., Marks, A., and DeLeo, J. A. (2006). Role of astrocytic S100 $\beta$ in behavioral hypersensitivity in rodent models of neuropathic pain. Neuroscience 140, 1003-1010. doi: 10.1016/j.neuroscience. 2006.02.070

van Elswijk, G., Kleine, B. U., Overeem, S., and Stegeman, D. F. (2007). Expectancy induces dynamic modulation of corticospinal excitability. J. Cogn. Neurosci. 19, 121-131. doi: 10.1162/jocn.2007.19.1.121

Vidor, L., Torres, I. L., Medeiros, L., Dussán-Sarria, J., Dall'Agnol, L., Deitos, A., et al. (2014). Association of anxiety with intracortical inhibition and descending pain modulation in chronic myofascial pain syndrome. BMC Neurosci. 15:42. doi: 10.1186/1471-2202-15-42

Warmenhoven, F., van Rijswijk, E., Engels, Y., Kan, C., Prins, J., van Weel, C., et al. (2012). The Beck Depression Inventory (BDI-II) and a single screening question as screening tools for depressive disorder in Dutch advanced cancer patients. Support. Care Cancer 20, 319-324. doi: 10.1007/s00520-010-1082-8

Werhahn, K. J., Kunesch, E., Noachtar, S., Benecke, R., and Classen, J. (1999). No title. J. Physiol. 517(Pt 2), 591-597. doi: 10.1111/j.1469-7793.1999.0591t.x

Wolfe, F., Clauw, D. J., Fitzcharles, M.-A., Goldenberg, D. L., Hauser, W., Katz, R. S., et al. (2011). Fibromyalgia criteria and severity scales for clinical and epidemiological studies: a modification of the ACR preliminary diagnostic criteria for fibromyalgia. J. Rheumatol. 38, 1113-1122. doi: 10.3899/jrheum. 100594

Wolfe, F., Smythe, H. A., Yunus, M. B., Bennett, R. M., Bombardier, C., Goldenberg, D. L., et al. (1990). The American College of Rheumatology 1990 criteria for the classification of fibromyalgia. report of the multicenter criteria committee. Arthritis Rheum. 33, 160-172. doi: 10.1002/art.17803 30203

Woolf, C. J. (2011). Central sensitization: implications for the diagnosis and treatment of pain. Pain 152(Suppl.), S2-S15. doi: 10.1016/j.pain.2010.09.030 
Yunus, M. B. (2007). Fibromyalgia and overlapping disorders: the unifying concept of central sensitivity syndromes. Semi. Arthritis Rheum. 36, 339-356. doi: 10. 1016/j.semarthrit.2006.12.009

Zanette, S. A., Dussan-Sania, J. A., Souza, A., Deitos, A., Torres, I. L. S., and Caumo, W. (2014). Higher serum S100B and BDNF levels are correlated with a lower pressure-pain threshold in fibromyalgia. Mol. Pain 10:46. doi: 10.1186/ 1744-8069-10-46

Ziemann, U. (2004). TMS and drugs. Clin. Neurophysiol. 115, 1717-1729. doi: 10.1016/j.clinph.2004.03.006

Ziemann, U., Lönnecker, S., Steinhoff, B. J., and Paulus, W. (1996a). Effects of antiepileptic drugs on motor cortex excitability in humans: a transcranial magnetic stimulation study. Ann. Neurol. 40, 367-378. doi: 10.1002/ana. 410400306

Ziemann, U., Lönnecker, S., Steinhoff, B. J., and Paulus, W. (1996b). The effect of lorazepam on the motor cortical excitability in man. Exp. Brain Res. 109, $127-135$.
Ziemann, U., Reis, J., Schwenkreis, P., Rosanova, M., Strafella, A., Badawy, R., et al. (2015). TMS and drugs revisited 2014 Clin. Neurophysiol. 126, 1847-1868. doi: 10.1016/j.clinph.2014.0 8.028

Conflict of Interest Statement: The authors declare that the research was conducted in the absence of any commercial or financial relationships that could be construed as a potential conflict of interest.

Copyright (c) 2018 Deitos, Soldatelli, Dussán-Sarria, Souza, da Silva Torres, Fregni and Caumo. This is an open-access article distributed under the terms of the Creative Commons Attribution License (CC BY). The use, distribution or reproduction in other forums is permitted, provided the original author(s) and the copyright owner(s) are credited and that the original publication in this journal is cited, in accordance with accepted academic practice. No use, distribution or reproduction is permitted which does not comply with these terms. 